\title{
Closed braids and knot holders associated to some laser dynamical systems: A pump-modulated Nd-doped fiber laser
}

\author{
E. A. Elrifai and Nazek A. AL-Essa \\ Princess Nourah Bint Abdulrahman University, Kingdom of Saudi Arabia: \\ 1- Department of Mathematical Sciences, College of Science. \\ 2- Deanship of Scientific Research. \\ eaelrifai@pnu.edu.sa \\ nazekaa@hotmail.com
}

\begin{abstract}
In this work the arising knots and links for the pump-modulated Nd-doped fiber laser is investigated. For the associated templates, some of their topological invariants, such as braid linking matrix, braid words, crossing number and linking number, are studied. It is recognized that the derived topological invariants are quietly dependent on the control parameters Using the tools of the braid theory, it is shown that pump-modulated Nd-doped fiber laser knots and links are positive fibered knots and links.
\end{abstract}

Keywords: laser dynamics; knots and links; braid groups, symbolic dynamics, topological invariants.

Mathematics Subject Classification 2000: 37D45, 37B10, $20 \mathrm{~F} 36$.

\section{Introduction}

\section{$1.1 \quad$ Laser dynamics}

The word laser is an acronym taken from the phrase "light amplification by stimulated emission of radiation". Lasers are virtually everywhere around us, for example, optical data storage, optical telecommunication and medical applications. One of the most benefits of the laser regimes is its use as a test-bed for generating deterministic chaotic signals [1].

The exponential growth of the radiation intensity $I(t)$ inside a laser cavity has the general form $I(t)=I_{0} e^{\left[\left(y_{m}-y_{c}\right) t\right]}$, where $y_{m}$ and $y_{c}$ are the cavity growth and decay rates, respectively. They are given by $y_{m}=2 \alpha_{m} L_{m} / t_{r t}$ and $y_{c}=\left[2 \alpha_{0} L+\ln \left(1 / R_{t o t}\right)\right] / t_{r t}$, where $\alpha_{m}$ is the gain coefficient of the active medium, $\alpha_{0}$ is the cavity scattering loss coefficient, $L$ is the overall cavity length, $L_{m}$ is the length of the active medium, $R_{\text {tot }}$ is the product of the reflectivity of the cavity mirrors, and $t_{r t}$ is the cavity round-trip time. While the growth equation is $d I(t) / d t=\left[y_{m}(t)-y_{c}(t)\right] I(t)$. But the laser dynamical systems has two major advantages over fuid systems, the ...rst one is that the time scales intrinsic to a laser are much shorter than the time scales for fluid experiments, it is from $10^{-7}$ to $10^{-3} \mathrm{sec}$. While the second difference is that the reliable laser models exist in terms of a small number of ordinary differential equations whose solutions show close qualitative similarity to the behavior of the lasers that are modeled [2].

Under modulation of a control parameter, it is known that this laser experiment exhibits chaotic oscillations. They modulate the pump parameter $A(t)=A_{0}(1+m \cos \omega t)$ around the average value $A_{0}=2.7$ with a rate $m=0.6$. The frequency is chosen as a control parameter and swept from to the relaxation frequency $\omega_{r}$, which under these conditions is about $36 \mathrm{kHz}$. Also they have a sequence of chaotic windows $C_{1 / 2}, C_{1 / 3}$ and $C_{1 / 4}$ located around $\omega_{1 / 2} \approx 18 \mathrm{kHz}, \omega_{1 / 3} \approx 12 \mathrm{kHz}$ and $\omega_{1 / 4} \approx 9 \mathrm{kHz}$. respectively. In fact these notations has been chosen to emphasize the harmonic relation between the chaotic frequencies and $\omega_{r} \approx 2 \omega_{1 / 2} \approx 3 \omega_{1 / 23} \approx 4 \omega_{1 / 4}$ they also registered no chaotic behavior around $\omega_{r}$. In the templates associated to this fiber laser system, it is observed that the global torsion increases systematically from one tongue to the next, as in figure 1, [2]. 

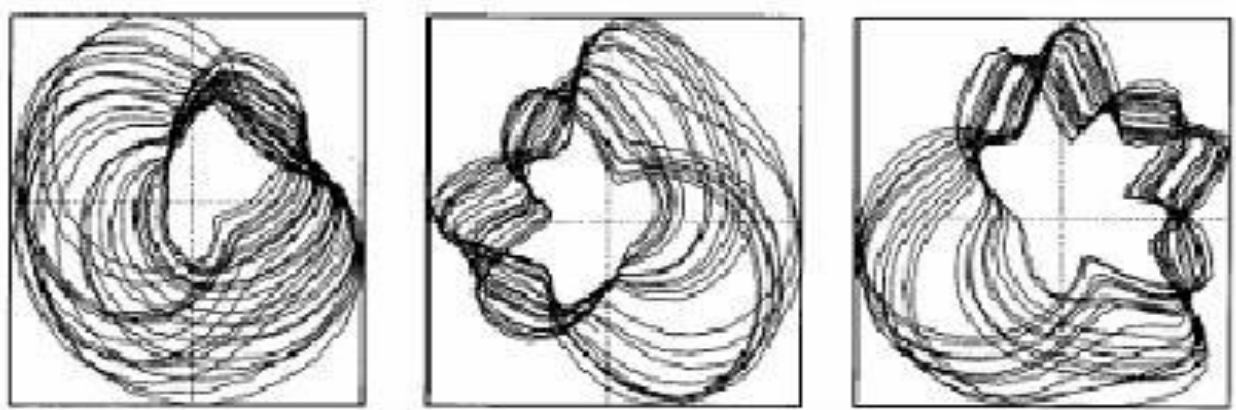

Fig. 1: Some of the templates observed in the fiber laser experiment.

\section{$1.2 \quad$ Knots, links and braids}

Knot theory studies the embedding of one dimensional smooth manifolds with non-self-intersecting in a three dimensional spaces. Any knot has infinite different plane projections in $\mathbb{R}^{2}$. A projection is regular if the set of selfintersections consists only of a finite number of transverse double points. Knot and link theory and its applications is very interesting and has many applications [3]. Figure. 2 gives different knot and link types.
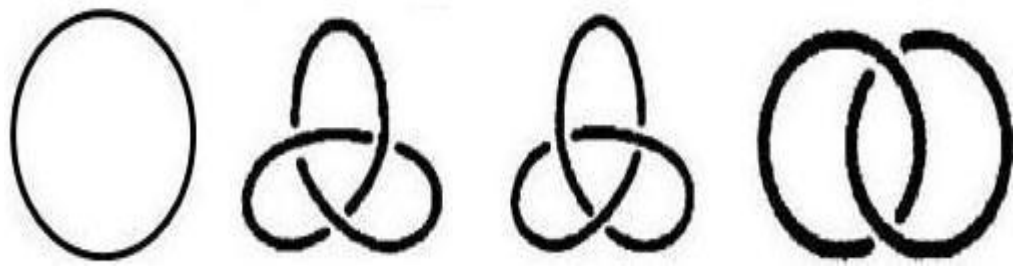

Fig. 2: From left to right, the unknot, the trefoil knot, the figure eight knot and the Hopf link.

Braid theory is very useful in studying knot theory since any oriented knot can be represented as a closed braid. A geometric braid is presented by two horizontal lines each with $n$ base points and $n$ strands from upper points to lower points, as in Fig. 3a. By connecting opposite ends of the strands we form a closed braid. Each closed braid is a knot or link, and conversely, as in Fig. 3b. In fact braid groups allow us to pass back and forth between geometric study on knots and algebraic study of braids [4]. In an algebraic point of view, the braid group $B_{n}$ has a group presentation

$$
B_{n}=\left\{\sigma_{i}, i=1,2, \ldots, n-1 \mid \begin{array}{l}
\sigma_{i} \sigma_{i+1} \sigma_{i}=\sigma_{i+1} \sigma_{i} \sigma_{i+1}, i=1,2, \ldots, n-2 \\
\sigma_{i} \sigma_{j}=\sigma_{j} \sigma_{i},|i-j| \geq 2
\end{array}\right\}
$$

where the generators $\sigma_{i}$ and their inverses $\sigma_{i}^{-1}$ can be represented geometrically as illustrated in Fig.3a. A braid is positive if all its crossings are positive according to the orientation from upper line to lower line [4].

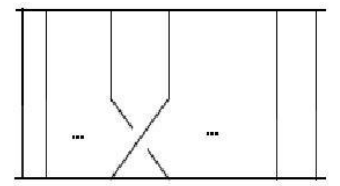

$12 \ldots i \quad i+1 \cdots n-1 n$

$\sigma_{i}$

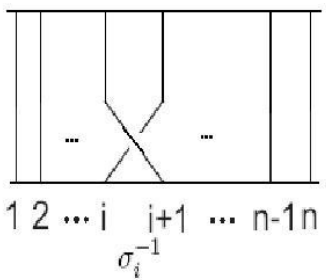

Fig. 3: (a) The braid generators

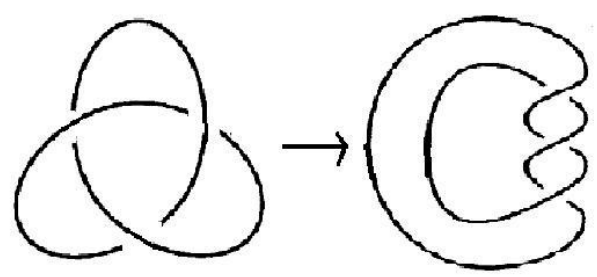

(b) Trefoil knot as a closed braid

A braid is said to be positive if it can be written as a product of elements $\sigma_{i}^{k}, k \in \mathbb{N}$ without involving negative powers. A positive braid is called a positive permutation braid if each pair of its strings cross in a positive sense at most once. The braid in which any two strings cross each other exactly once in a positive sense is called the 
fundamental braid, denoted $\Delta_{n}$, with

$$
\begin{aligned}
\Delta_{1} & =i d ., \Delta_{2}=\sigma_{1}, \Delta_{3}=\sigma_{1} \cdot \sigma_{2} \sigma_{1}, \Delta_{n}=\Delta_{n-1} \sigma_{n-1} \sigma_{n-2} \ldots \sigma_{2} \sigma_{1} \\
\Delta_{n} & =\left(\sigma_{1}\right)\left(\sigma_{2} \sigma_{1}\right) \ldots\left(\sigma_{n-2} \ldots \sigma_{2} \sigma_{1}\right)\left(\sigma_{n-1} \sigma_{n-2} \ldots \sigma_{2} \sigma_{1}\right) \\
& =\left(\sigma_{1} \sigma_{2} \ldots \sigma_{n-1}\right)^{n}
\end{aligned}
$$

In fact, every element $\beta$ in $B_{n}$ can be represented by a unique word $\beta=\Delta^{2 r} P_{1} P_{2} \ldots P_{s}, r \in \mathbb{Z},|r|$ is maximal, $s$ is minimal and each $P_{i}$ is the longest possible positive permutation braid for all such representations, such this form is called the left canonical form [5].

\subsection{Dynamical systems, topological invariants and symbolic dynamics}

The existence and uniqueness solutions of differential equations is the relevant of the relation between knot theory and dynamical systems. In fact, closed periodic orbits in a three dimensional manifold flow are just an embedding of a finite collection of one dimensional manifolds. So it is the knot and link theory, and it is the consideration of topological properties of closed orbits as knots and links. In general there are different types of invariants, such as metric invariants, dynamical invariants and topological invariants. Here we are interested with topological invariants, linking numbers, relative rotation rate and the structure of templates (knot holders).

The template is a compact 2-dimensional manifold with boundary and smooth expanding semi-flow built from a finite number of branch line charts and the semi-flow is tangent to the rest of the boundary. The invariant set of a template is the set of orbits of the semi-flow that never exit. Lorenz template is a simple and interesting example for templates, figure 4. A Lorenz braid is any finite set of braid strands that embeds on the Lorenz template.

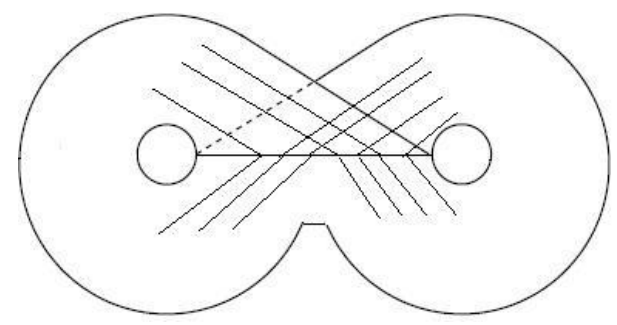

Fig. 4: Lorenz template

For algebraic invariants of knot holders, label the top of the branches of the knot holder from left to right $0,1,2, \ldots, k-11$, also arrange the knot holder so that at the bottom of the branches, start ordering back to front from left to right, which called the standard insertion. Hence, the braid linking matrix of a knot holder with $k$ branches is a square symmetric $k \times k$ matrix $B=\left(b_{i j}\right)$, where $b_{i j}$ is the sum of half twists in the $\mathrm{i}^{\text {th }}$ branch (the local torsion of the ith branch), and $b_{i j}$ is the sum of the crossings between the $\mathrm{i}^{\text {th }}$ and the $\mathrm{j}^{\text {th }}$ branches of the ribbon graph with standard insertion. So that $b_{i j}$ is twice the linking number $l k_{i j}$ of the ribbon graph for the $\mathrm{i}^{\text {th }}$ and $\mathrm{i}^{\text {th }}$ branches. A branch of a template is called orientation preserving if the local torsion (the number of half twists) is an even integer, and a branch is called orientation reversing if the local torsion is an odd integer, [6]. Examples are in figure 5.

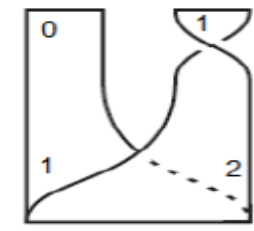

$B=\left[\begin{array}{cc}0 & 0 \\ 0 & -1\end{array}\right]$
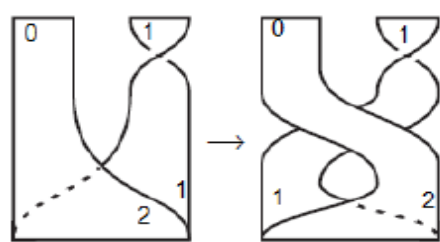

$B=\left[\begin{array}{cc}0 & -1 \\ -1 & 1\end{array}\right]$

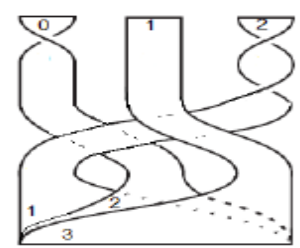

$B=\left[\begin{array}{ccc}-1 & 0 & 1 \\ 0 & 0 & -1 \\ 1 & -1 & 2\end{array}\right]$

Fig. 5: Knot holders with their associated braid linking matrices. 
Algorithm 1 The symbolic name of a knot on a template is given by recording the branches over which the knot passes. Now consider a template of $k$ branches, then we are going to construct a $k$-ary tree which encodes the ordering of points on the orientation preserving and reversing branches of the template. The steps of the algorithm are as follows [6]:

- At the level $n=1$, write the symbolic names for the branches from left to write as $0,1,2, \ldots, k-1$.

- At the second level, $n=2$, we start by recording the symbolic names at the 1 st level of the tree according to the ordering rule: if the ith branch of the template is orientation preserving then we write the symbolic names in forward order $0,1,2, \ldots, k-1$, if the ith branch is orientation reversing then we write the symbolic names at the $1^{\text {st }}$ level in reverse order $k-1, k-2, \ldots, 2,1,0$.

- The $n+1$ level is constructed from the $\mathrm{n}^{\text {th }}$ level by the ordering rule: if the $\mathrm{i}^{\text {th }}$ symbol (branch) at the $\mathrm{n}^{\text {th }}$ level is orientation preserving then we record the ordering of the symbols found at the $\mathrm{n}^{\text {th }}$ level (read symbols from left to right as it is); if the $i^{\text {th }}$ symbol labels an orientation reversing branch, then we reverse the ordering of the symbols found at the $\mathrm{n}^{\text {th }}$ level (read symbols from right to left). Then The symbolic names for the three branches knot holder illustrated at the right in figure 5 , will be as in the following table.

\begin{tabular}{|c|c|c|}
\hline 0 & 1 & 2 \\
\hline 210 & 012 & 012 \\
\hline 210210012 & 210012012 & 210012012 \\
\hline 210210012210210012210012012 & 210210012210012012210012012 & 210210012210012012210012012 \\
\hline$\ldots$ & $\ldots$ & $\ldots$ \\
\hline \multicolumn{2}{|c|}{ Table1: The tree of symbols for the knot holder in the right of figure 5 } \\
\hline
\end{tabular}

Example 2 Finally, to find the knot from the above table of symbols, we have to read down the $k-$ ary tree. In figure 6, from right to left and up to period 3, the symbols 001,110,221 with colors blue, yellow and black, respectively, admits the associated braid word. Hence we can close the braid and has a link with three components.
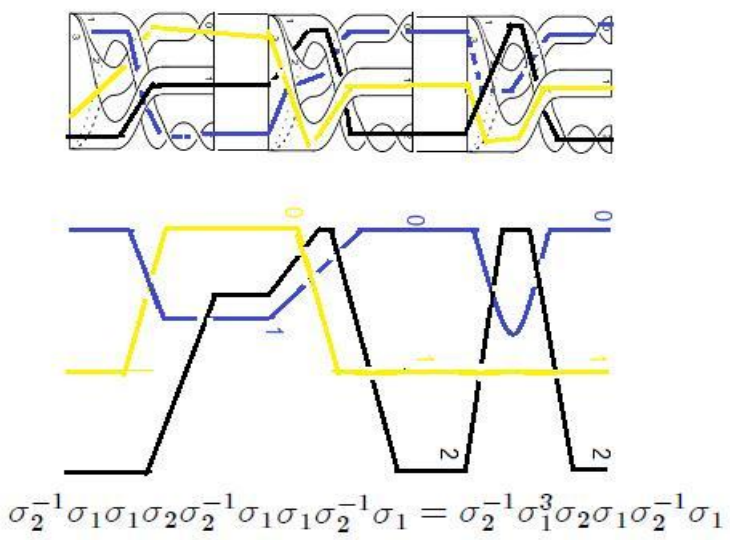

Fig. 6: A braid word from symbolic name

The braid in Fig. 6 has the equivalent braid words

$$
\sigma_{2}^{-1} \sigma_{1} \sigma_{1} \sigma_{2} \sigma_{2}^{-1} \sigma_{1} \sigma_{2} \sigma_{1} \sigma_{2}^{-1} \sigma_{1}=\sigma_{2}^{-1} \sigma_{1} \sigma_{1} \sigma_{1} \sigma_{2} \sigma_{1} \sigma_{2}^{-1} \sigma_{1}=\sigma_{2}^{-1} \sigma_{1} \sigma_{1} \sigma_{2} \sigma_{1} \sigma_{2} \sigma_{2}^{-1} \sigma_{1}=\sigma_{2}^{-1} \sigma_{1}^{2} \sigma_{2} \sigma_{1}^{2}
$$

\section{Knotted periodic orbits in pump-modulated Nd-doped fiber laser dynamics}

Now, back to the introduction where the templates associated to the pump-modulated Nd-doped fiber laser, and for various values of modulation frequency. There are a sequence of chaotic windows $C_{1 / 4}, n \in \mathbb{N}$, where the global torsion increases systematically from one tongue to the next, as in figure 1. In [1] a topological reorganization of the 
chaotic regimes as templates with two branches is given as in figure 7.
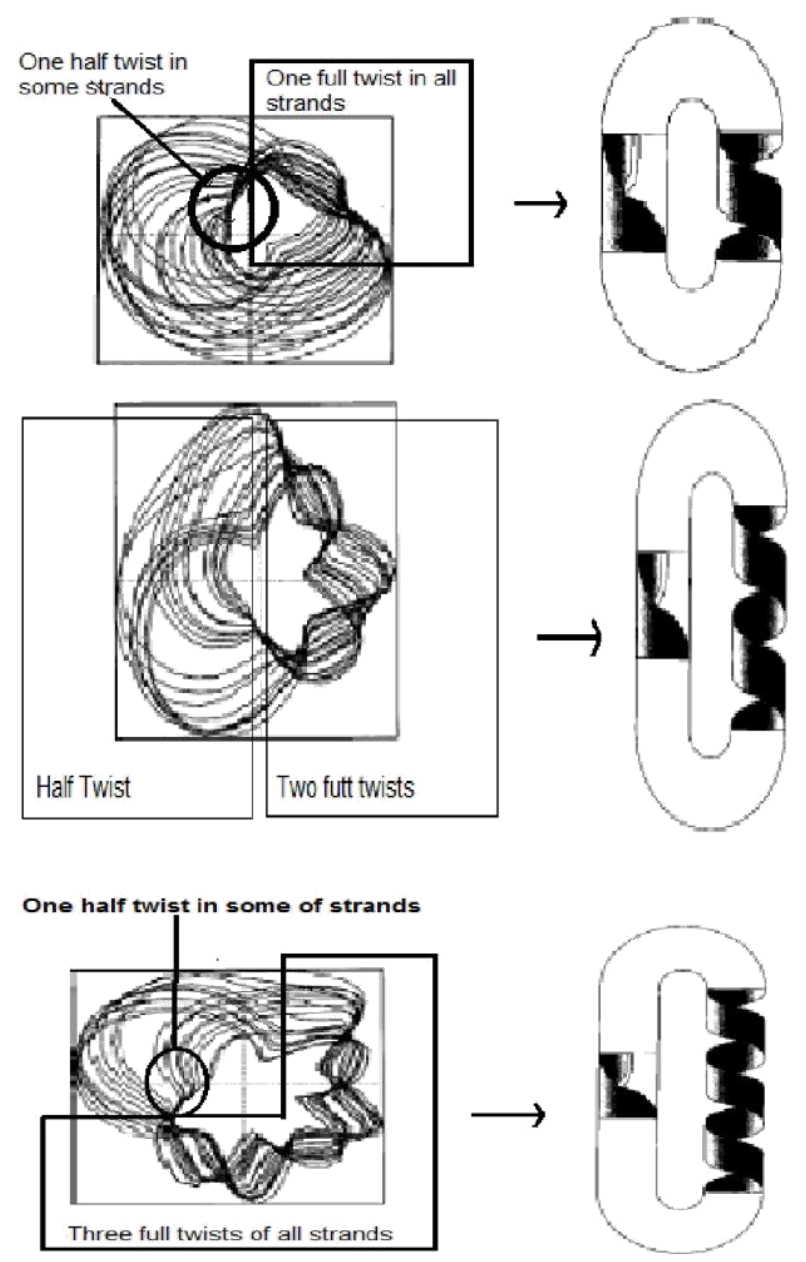

Fig. 7: Some attractors and the associated template

Proposition 3 The template associated to a modulated Nd-doped fiber laser, with the attractor in the subharmonic region $\omega_{1 / n}$ has the $2 \times 2$ braid linking matrix $B=\left(b_{i j}\right)$, where

$$
b_{11}=b_{12}=b_{21}=2(n-1), b_{22}=2(n-1)+1
$$

Proof. Take a strip of one half twist. Keep the top and bottom lines fixed, then cut the strip along the middle. This yields two twisted and linked ribbons with linking number equal one, while each ribbon has one full twist, as in figure 8a. Now let us consider the case of $\omega_{1 / 4}$, which illustrated in figure $8 \mathrm{~b}$, and cut it as in figure 8a. The resulting template is a branched 2-diminsional manifold with two layers, as in figure 8c. Each layer has three full twists and one of them has one more half twist, while both have linking number equal three. Which implies that the corresponding braid linking matrix has diagonal with $b_{11}=2(n-1), b_{22}=2(n-1)+1$ and the off-diagonal with $b_{12}=b_{21}=2(n-1)$. The general case has the braid linking matrix

$$
B=\left[\begin{array}{cc}
2(n-1) & 2(n-1) \\
2(n-1) & 2(n-1)+1
\end{array}\right]
$$

for the subharmonic region $\omega_{1 / 4}$ 
a

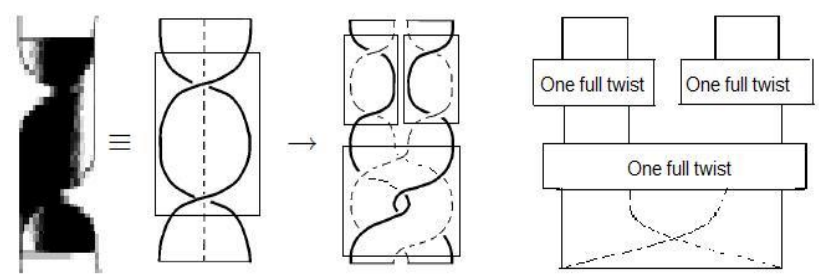

b
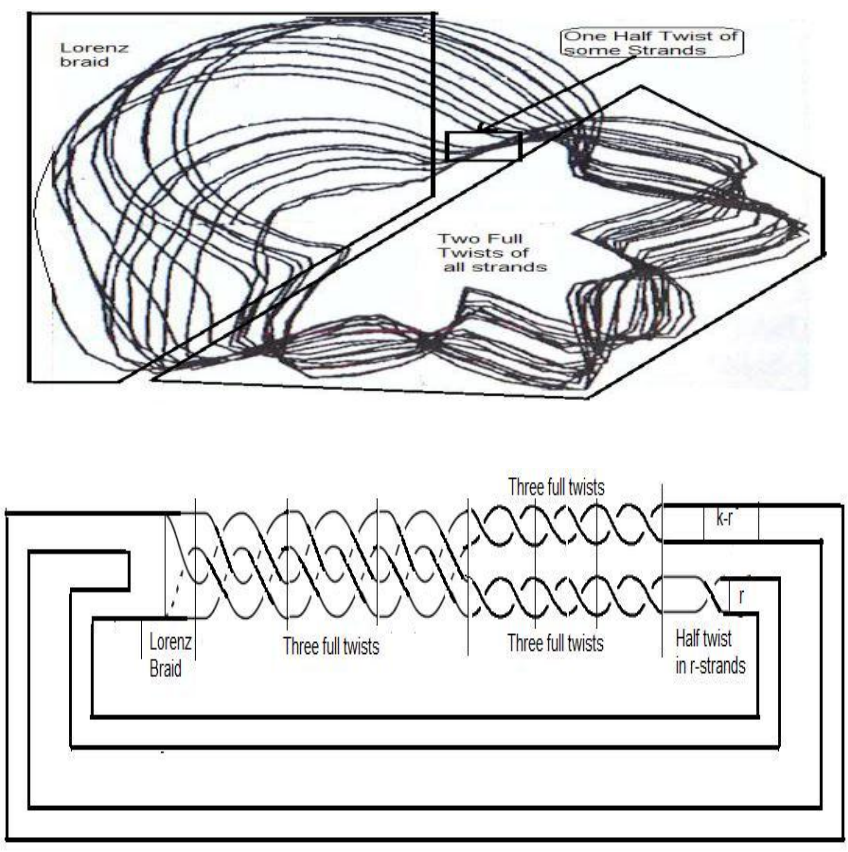

Fig. : 8: Cutting a ribbon with one full twist along the middle line

The above proposition provides a braid word associated to the trajectories in the general template in the subharmonic region $\omega_{1 / n}$.

Theorem 4 The closed periodic orbits associated to a modulated Nd-doped fiber laser, with the attractor in the subharmonic region $\omega_{1 / n}$ have the braid representatives

$$
\beta_{n, k, r}=\Delta_{k}^{2(n-1)} \Delta_{r} L(r, k-r), n, k, r \in \mathbb{N}, r<k
$$

Lemma 5 The braid representative $\beta_{n, k, r}=\Delta_{k}^{2(n-1)} \Delta_{r} L(r, k-r)$ of the orbits associated to a modulated $\mathrm{Nd}$-doped fiber laser is in the left canonical form.

Proof. The Lorenz word $L(r, k-r)$ is a positive braid word. So no self-intersection in each band of strands. Hence the word $P_{1}=\Delta_{r} L(r, k-r)$ is a one positive permutation braid. Then the braid $\beta_{n, k, r}=\Delta_{k}^{2(n-1)} P_{1}$ is in a left canonical form

Call such these braids $\beta$, by the Nd-doped fiber laser braids. And their associated closed braids $\hat{\beta}$, by the Nddoped fiber laser knots and links. The braid index $b(\hat{\beta})$ of a knot or link $\hat{\beta}$ is defined to be the minimal number of 
strands of a braid, whose closure is the knot or the link, $b(\hat{\beta})$ is always exists [3]. Positive knots are called the knots with diagrams of all crossings positive.

Corollary 6 The Nd-doped fiber laser knots and links are fibered with braid index $\mathrm{n}$.

Proof. Since the Nd-doped fiber laser braids are positive braids with full twists, then they are minimal braid representatives. So the associated closed braids are fibered knots and links, [7]

From theorem 2, and for different choices of the integers $n, k$ and $r$, we have infinite number of knots and links. For instance, if $n=2, k=2, r=1$ the braid

$$
\beta_{2,2,1}=\Delta_{2}^{2} \Delta_{1} L(1,1)=\sigma_{1}^{2} . i d ., \sigma_{1}=\sigma_{1}^{3}
$$

Then $\hat{\beta}_{2,2,1}$ is the trefoil knot. Also for $n=3, k=3, r=1$ the braid

$$
\beta_{3,3,1}=\Delta_{3}^{4} \Delta_{1} L(1,2)=\left(\sigma_{1} \sigma_{2}\right)^{6} \sigma_{1} \sigma_{2}
$$

Then $\hat{\beta}_{3,3,1}$ is a link of two components.

\section{Conclusion}

We applied the knot theory invariants to the $\mathrm{Nd}$-doped fiber laser. According to the topological invariants (braid linking matrix, braid words, crossing number and linking number) such these orbits are studied. They are positive fibered knots and links. The system yields an infinitely many different closed periodic orbits. That is according to the change of the control parameters.

\section{Acknowledgements}

The authors would like to express their gratitude to the deanship of scientific research at Princess Nourah Bint Abdulrahman university for supporting and funding this work No. 37-s-171. The deanship of scientific research owns the intellectual property rights.

\section{References}

[1] C. Phipps "Laser Ablation and its Applications" Springer Series in Optical Sciences, Vol. 129, 2007.

[2] G. Boulant, M. Lefranc, S. Bielawski and D. Derozier "Horseshoe templates with global torsion in a driven laser". Physical Review E, Vol. 55, No. 5, 5082-5091, 1997.

[3] K. Murasugi "Knot theory and its applications", Birkhauser Boston, 1996.

[4] Kassel, Christian, Turaev, Vladimir "Braid Groups" " Graduate Texts in Math-ematics, Vol. 247, 2008.

[5] E. A. Elrifai and H. Morton "Algorithms for positive braids" Quart. J. Math. Oxford (2), 45 (1994), 479-497.

[6] N.B. Tufillaro, T. Abbott and J. reilly "An experimental approach to nonlinear dynamics and chaos" , Reading, MA: Addison Wesley, 1992.

[7] A. Stoimenowon "Crossing number of positive knots and braids and braid index criteria of Jones and MortonWilliams-Franks" , Transactions of the AMS , Volume 354, Number 10, 3927-3954, 2002. 